\title{
A search for distant, pulsating red giants in the southern halo ${ }^{\star}$
}

\author{
N. Mauron ${ }^{1}$, K. S. Gigoyan ${ }^{2}$, T. R. Kendall ${ }^{3}$, and K. M. Hambleton ${ }^{4}$ \\ 1 Université de Montpellier, Laboratoire Univers et Particules de Montpellier CNRS-IN2P3, Place Bataillon, 34095 Montpellier, \\ France \\ e-mail: nicolas.mauron@umontpellier.fr \\ 2 NAS RA V.A. Ambartsumian Byurakan Astrophysical Observatory (BAO), Byurakan, Armenia \\ 318 Market Square, Northampton NN1 2DL, UK \\ 4 Department of Astrophysics and Planetary Science, Villanova University, 800 Lancaster Avenue, Villanova, PA 19085, USA
}

Received 28 October 2019 / Accepted 16 December 2020

\begin{abstract}
To investigate the asymptotic giant branch (AGB) population in the Galactic halo, we search for pulsating AGB stars at a heliocentric distance $D>50 \mathrm{kpc}$. Our research is based on the Catalina Southern Survey (CSS) catalogue of variables, comprising 1286 longperiod variables (LPVs) with $\delta<-20^{\circ}$. We first focus on the 77 stars in the cap $|b|>30^{\circ}$ for which spectral M-type or C-type classification can be derived from Hamburg-ESO objective prism spectra. Most of these are oxygen-rich (M-type) and very few are carbon rich. The periods are in the range 100-500 days, and CSS amplitudes are up to 3 mag. In this small sample, no halo AGB star is fainter than $K_{\mathrm{s} 0}=12.5$. This may be due to the scarcity of AGBs in the outer halo, or insufficient instrumental depth. Leaving aside spectral information, we then searched for even fainter pulsators $\left(K_{\mathrm{s}}>12.5\right)$ in the entire CSS catalogue. Gaia astrometry makes it possible to identify some contaminants. Our final result is the identification of ten candidate distant LPVs. If these ten stars obey the fundamental mode $K$-band period-luminosity relation used for Miras and small-amplitude Miras, their distances are between 50 and $120 \mathrm{kpc}$ from the Sun. In a diagram showing distance versus Gaia tangential velocity, these ten stars have positions consistent with that of other objects in the halo, such as globular clusters and dwarf galaxies. We detect some underluminous AGBs that deserve further study. Finally, the halo LPVs ressemble the slow redder variable of globular clusters when colour and periods are compared.
\end{abstract}

Key words. stars: late-type - Galaxy: halo

\section{Introduction}

The stellar populations located in the Galactic halo contain information about the general properties of the Galaxy, such as its mass, formation and evolution (Helmi 2008; Bland-Hawthorn \& Gerhard 2016; Deason et al. 2021). They are also essential for comparing the diverse halos of similar galaxies (Monachesi et al. 2016; Harmsen et al. 2016). Large and recent surveys use RR Lyr variable stars (Hernitschek et al. 2016; Sesar et al. 2017; Stringer et al. 2021), blue horizontal branch stars (Thomas et al. 2018; Starkenburg et al. 2019), K-type giants (Xue et al. 2015; Janesh et al. 2016; Yang et al. 2019), or M-type giants (Bochanski et al. 2014). In this paper we focus on the population of evolved long-period variables (LPV). We follow previous works by Huxor \& Grebel (2015) centred on carbon stars, by Grady et al. (2019) who used LPVs as age indicators in the Galactic components and as tracers of merging debris, and Mauron et al. (2019a,b) who studied M-type LPVs in the northern halo and Sagittarius tidal arms.

Long-period variables evolve along the asymptotic giant branch (AGB) and deserve attention because they are more luminous than some of the tracers cited above. Miras or smallamplitude Miras are rarer than giants by more than an order of magnitude, but, like RR Lyr, follow the period-luminosity (PL)

\footnotetext{
* A detailed catalogue of the 77 high-latitude $\mathrm{M}$ or $\mathrm{C}$ stars (Full Table 5) is only available at the CDS via anonymous ftp to cdsarc.u-strasbg.fr (130.79.128.5) or via http://cdsarc. u-strasbg.fr/viz-bin/cat/J/A+A/650/A146
}

relation. There are old LPVs in many globular clusters (GCs) with periods in the range of $\sim 30-250$ days. If the nascent Galaxy contained a large number of now completely dissolved GCs (Gnedin \& Ostriker 1997; Odenkirchen et al. 2001; Lee et al. 2004), one might expect a fossil population of cluster LPVs in the halo. Similar and more numerous LPVs can also originate from dislocated dwarf galaxies.

The old AGB populations in GCs are M-type. These cluster stars are in most cases semi-regulars (SRa or SRb type), or, more rarely, Miras possessing a large flux amplitude $\Delta V>2.5 \mathrm{mag}$ (Clement 2001, 2017). The halo AGB population also comprises cool N-type carbon (C) stars that have intermediate ages; from the census of halo C stars of Huxor \& Grebel (2015), there are about 40 stars at more than $50 \mathrm{kpc}$ from the Sun, with 9 at more than $100 \mathrm{kpc}$. Roughly two-thirds of cool N-type C stars belong to the Sgr stream. Concerning the M stars, Mauron et al. (2019b) presented a catalogue covering the section of sky from $\delta>-20^{\circ}$. These latter authors found 57 stars at $D>50 \mathrm{kpc}$ and 6 at $>$ $100 \mathrm{kpc}$; again, about two-thirds of them are in the Sgr stream.

Here, our goal is to increase the known sample of LPVs with $D>50 \mathrm{kpc}$ by examining the complementary region with $\delta<-20^{\circ}$. We use the Catalina Southern Survey (CSS) LPV catalogue of Drake et al. (2017), and separate the M-type from C-type stars by exploiting objective prism plates. Attention was paid to contaminants: in Mauron et al. (2019a), we discovered that numerous faint and distant stars are due to instrumental artefacts. Thanks to the Gaia EDR3 data, some supplementary contaminating stars can be discarded. After analysing the data in Sect. 2, we discuss our results in Sect. 3. We finally conclude 
that very few LPVs are as distant as $50 \mathrm{kpc}$ in the CSS survey, and only ten distant candidates are found.

\section{Analysis}

The CSS catalogue contains 1286 LPVs distributed over an area of $\sim 10000$ square degrees at a declination of $\delta<-20^{\circ}$. In particular, it provides periods $P$, time-averaged magnitudes $m_{\mathrm{CSS}}$, and amplitudes $\Delta m_{\mathrm{CSS}}$, based on observations made from 2005 to 2013. Its limiting magnitude is $m_{\mathrm{CSS}} \sim 17$. The CSS instrument has no filter, and for red stars similar to those of this work, $m_{\mathrm{CSS}}$ is close to a $I$-band magnitude. A majority of these 1286 LPVs are in the Galactic disc, with very few at high Galactic latitude, as shown in Fig. 1 of Mauron et al. (2019a). Also, a fraction of the halo of the Large Magellanic Cloud is covered, and a number of LPVs are detected in the Fornax and Sculptor dwarf galaxies located at distances of $\sim 140$ and $90 \mathrm{kpc}$, respectively (van den Bergh 2000). This shows that the CSS catalogue is a suitable resource for detecting very distant LPVs $(D>50 \mathrm{kpc})$.

Of the 1286 CSS LPVs, 93 have been spectroscopically classified by exploiting the Hamburg-ESO objective prism survey (HES, Christlieb et al. 2004). Of these 93 stars, 16 are false variables due to a polluting Mira (their list is in Sect. 2). In Sect. 2.1, we focus on the remaining 77 stars and analyse their properties. In Sect. 2.2, we proceed by considering objects too faint to be classified with HES. Finally, in Sect. 2.3, we extend our search over the whole CSS LPV catalogue. The astrometric data that we use are mostly from the Gaia EDR3 catalogue.

\subsection{M-type and C-type stars from the CSS catalogue and the Hamburg-ESO survey}

Figure 1 shows a colour-magnitude diagram for stars that could be classified $\mathrm{M}$ or $\mathrm{C}$ on HES plates. The interstellar $E_{B-V}$ colour excess from Schlafly \& Finkbeiner (2011) is used to take into account Galactic extinction ${ }^{1}$. More precisely, we adopt $A_{V}=3.10 E_{B-V}, A_{J}=0.87 E_{B-V}$, and $A_{\mathrm{Ks}}=0.35 E_{B-V}$. We consider that all extincting dust is located between the Sun and the sample star, because most of the LPVs are much higher above the Galactic plane than the thickness $(\sim 100 \mathrm{pc})$ of the Galactic dust layer. It can be seen that M-type stars largely dominate our sample. Stars belonging to the Large Magellanic Cloud (LMC), Fornax, or Sculptor are indicated. For one object, KG-14 in the $\mathrm{LMC}$, it is not clear whether the spectrum is C or M-type, but its colour favours a carbon type that we adopt here. One can see that a small number of carbon-rich LPV stars are detected over the imprint of the HES plates. Table 1 lists their 2MASS identifications and main properties.

In Fig. 1, faint stars $\left(K_{\mathrm{s}}>8\right)$ appear slightly bluer than brighter stars, indicating that our sample is not homogeneous. This inhomogeneity is reinforced by the diagram of Fig. 2, where CSS amplitude is plotted versus period. There is first a main group with period from $\sim 100$ to 300 days, and amplitude $\Delta m_{\mathrm{CSS}}$ rising from 0.6 to $\sim 3 \mathrm{mag}$. Most of the $\mathrm{C}$ stars are slightly to the right of this group. A second family of 10 LPVs exist with $P>220$ days and relatively smaller amplitude $\left(\Delta m_{\mathrm{CSS}}<0.8 \mathrm{mag}\right)$ compared to objects of the main group. We show later that this large-period small-amplitude group is also separated with a colour based on Gaia and 2MASS photometry.

\footnotetext{
1 The colour excess is provided by the NASA/IPAC Galactic Dust Reddening and Extinction service at https://irsa.ipac. caltech. edu/applications/DUST
}

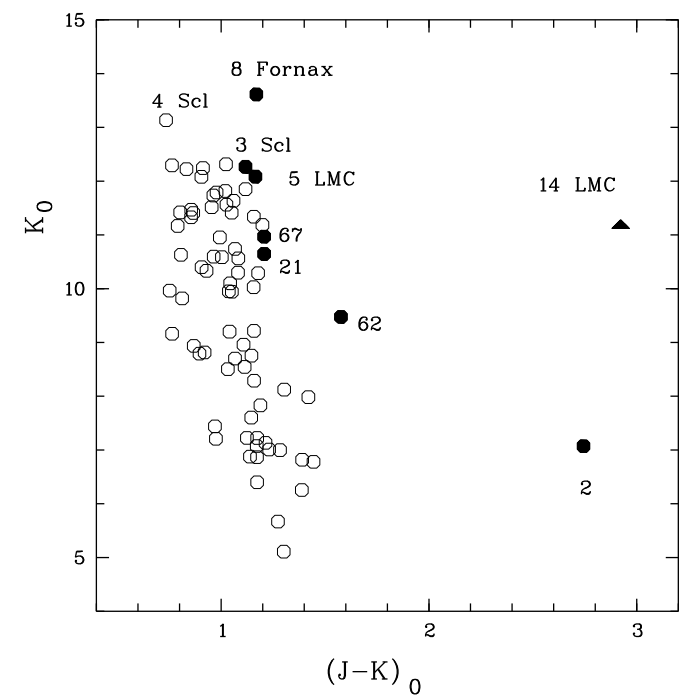

Fig. 1. Near-infrared colour-magnitude diagram of spectrally classified stars, showing the dereddened $K_{\mathrm{s} 0}$ magnitude as a function of $\left(J-K_{\mathrm{s}}\right)_{0}$ from 2MASS photometry. Uncertainties are comparable to symbol sizes. Red objects are to the right, faint objects at the top. Circles: M-type stars. Filled symbols: carbon stars. The labels correspond to the KG number: KG-2, 21, 62, 67 are in the halo. Objects labelled ' $3 \mathrm{Scl}$ ' and ' $4 \mathrm{Scl}$ ' are in Sculptor. The spectrum of KG-14 (filled triangle) is unclear, but its colour favors a carbon type.

Table 1. Properties of the stars labelled in Fig. 1.

\begin{tabular}{lcccccc}
\hline \hline KG & 2MASS name & $m_{\text {CSS }}$ & $P$ & $K_{\mathrm{S}}$ & Sp. & \\
\hline 2 & $00165576-4400406$ & 14.71 & 440 & 7.07 & $\mathrm{C}$ & halo \\
3 & $00595893-3328351$ & 16.23 & 196 & 12.27 & $\mathrm{C}$ & Scl \\
4 & $01012084-3353047$ & 16.18 & $91:$ & 13.14 & $\mathrm{M}$ & Scl \\
5 & $01015347-6518233$ & 15.71 & 162 & 12.09 & $\mathrm{C}$ & LMC \\
8 & $02391532-3415083$ & 17.71 & 196 & 13.62 & $\mathrm{C}$ & For \\
14 & $04360427-6347499$ & 17.76 & 324 & 11.21 & $\mathrm{C}$ & LMC \\
21 & $04563115-3129327$ & 14.26 & 147 & 10.65 & $\mathrm{C}$ & halo \\
62 & $21271642-3051573$ & 14.29 & 233 & 9.49 & $\mathrm{C}$ & halo \\
67 & $21434114-3414311$ & 14.78 & 158 & 10.98 & $\mathrm{C}$ & halo \\
\hline
\end{tabular}

Notes. We list the KG number, 2MASS identification, Catalina CSSband mean magnitude, period $P$ (in days), $K_{\mathrm{s}}$ magnitude, spectral classification and membership. The spectral type of KG-14 is uncertain on HES plates, but its colour favors a $\mathrm{C}$ type, adopted here. The last column gives membership. The coordinates of KG-62 are erroneous in Mauron et al. (2019a).

Below, we first comment on the $\mathrm{C}$ stars, and discuss later on the more numerous $M$ stars.

\subsubsection{C stars}

The brighter C-type star, KG-2, has a 2MASS single-epoch magnitude $K_{\mathrm{s}}=7.07$, a period of $P=440$ days, and peak-to-peak $m_{\mathrm{CSS}}$-band amplitude $\Delta m_{\mathrm{CSS}}=2.5 \mathrm{mag}$. This star is HG-2 in the catalogue of Huxor \& Grebel (2015). Neither the origin nor the metallicity is known for this star located at high Galactic latitude $\left(b=-71^{\circ}\right)$. If this star is similar to solar-metallicity, cool C stars of the solar neighbourhood, with a Gaia-based distance, then $M_{\mathrm{Ks}} \approx-8.5 \pm 0.8$ from Fig. 11 of Abia et al. (2020). If it is similar to carbon stars of the more metal-poor Small Magellanic 
N. Mauron et al.: Searching cool pulsating giants in the Galactic halo

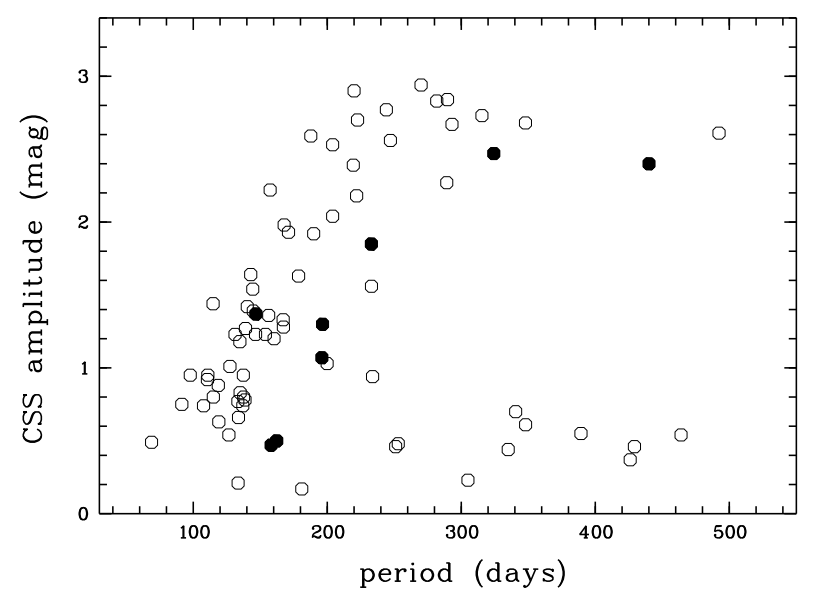

Fig. 2. Catalina amplitude $\Delta m_{\mathrm{Css}}$ versus period. Circles: M-type stars. Filled circles: carbon stars. The two stars in the upper right-hand corner are the halo carbon star KG-2 $\left(K_{\mathrm{s}}=7.1, D \sim 14 \mathrm{kpc}\right.$, discussed in the text) and the M-type star WOH S24 (alias KG-15; $K_{\mathrm{s}}=10.0$ at $51 \mathrm{kpc}$ ), located in the Large Magellanic Cloud.

Cloud (SMC), we obtain a very similar estimate, $M_{\mathrm{Ks}} \approx-8.60$ by applying the period-luminosity relation ${ }^{2}$.

Consequently, we find the distance of KG-2 from the Sun and from the Galactic plane to be $D=14 \mathrm{kpc}$. Concerning the uncertainty on $D$, we have $D=10^{* * 0} 0.2\left(K_{\mathrm{s}}-M_{\mathrm{Ks}}+5\right)$ and errors on $M_{\mathrm{Ks}}$ and $K_{\mathrm{s}}$ have to be considered. The first is typically $\sigma_{M_{\mathrm{Ks}}}=0.15 \mathrm{mag}$ (i.e. the dispersion of the PL relation; Whitelock et al. 2009, their Table 2). The second originates from the difference between the required but unknown time-average $K_{\mathrm{s}}$-band flux and the available single-epoch 2MASS photometry. This difference is $\delta K_{\mathrm{s}}=0.2 \Delta m_{\mathrm{CSS}}+0.08 \mathrm{mag}$ (see details in Mauron et al. 2019b). Adding these errors in quadrature leads to a relative error on $D$ of $9 \%$ for $\Delta m_{\mathrm{CSS}}=0.2$, to $30 \%$ for $\Delta m_{\mathrm{CSS}}=2.5$. Therefore, the distance of KG-2 is $14 \pm 4 \mathrm{kpc}$. This distance is larger than the one derived by Huxor \& Grebel $(11 \pm 4)$ because they use the Wesenheit indices and consequently take into account circumstellar extinction better for this very red star. KG-2 is angularly located far from the lane of $\mathrm{C}$ stars in the Sgr arms and its origin is unclear. One possibility is that it may have been ejected from the Galactic disc, as found by Grady et al. (2019) for some Miras.

The $\mathrm{C}$ stars KG-21, KG-62, and KG-67 are warm, that is $\left(m_{\mathrm{CSS}}-K_{\mathrm{s}}\right)_{0} \sim 3.5-4.8$, compared to KG-2 with $\left(m_{\mathrm{CSS}}-K_{\mathrm{s}}\right)_{0} \sim$ 7.6. They are short-period variables (see Table 1) and have $K_{\mathrm{s}} \approx 9-11$, which is fainter than KG-2. While KG-62 is named HG-108 in the census of Huxor \& Grebel (2015), KG-21 and KG-67 are not in the list of these latter authors. If they are members of the Sgr tidal arms, their $[\mathrm{Fe} / \mathrm{H}]$ is probably between -1.2 to -0.5 , which is the range of metallicity in the Sgr arms (see Fig. 2 of Carlin et al. 2018). Because the SMC has a mean average abundance $[\mathrm{Fe} / \mathrm{H}] \sim-0.73$ (van den Bergh 2000) or $\sim-1.0$ (McConnachie 2012), our best choice is to adopt again the

2 Soszyński et al. (2007) provides for SMC C-stars of sequence $\mathrm{C}$ the following relation $M_{\mathrm{Ks}}=-7.15-4.22 \log _{10}(P / 200)$, where $P$ is in days, $K_{\mathrm{S}}$ is the time-averaged magnitude determined using $I$-band light curves (Sect. 3 of their paper), and where a SMC distance modulus of 18.99 is used. We note that, throughout this paper, we assume all stars (M-type or C-type) to lie on the pulsation sequence C. Their distances would increase by a factor 1.8 if they were to lie on the more luminous sequence C'. For more details, see Table 2 and Figs. 3 and 4 of Soszyński et al. (2007).
Table 2. Sixteen false LPV objects.

\begin{tabular}{lcccccc}
\hline \hline Star & $\alpha$ & $\delta$ & Mira & $\theta$ & $P_{\text {KG }}$ & $P_{\text {Mira }}$ \\
\hline KG-22 & 85.43000 & -38.9572 & SS Col & 26 & 364 & 358 \\
KG-36 & 192.93978 & -26.7755 & EP Hya & 23 & 168 & 168 \\
KG-42 & 207.26013 & -28.5105 & W Hya & 514 & 400 & 389 \\
KG-41 & 207.25596 & -28.4919 & W Hya & 447 & 397 & 389 \\
KG-44 & 209.18330 & -25.5373 & FT Hya & 32 & 214 & 216 \\
KG-45 & 209.18796 & -25.5651 & FT Hya & 72 & 214 & 216 \\
KG-46 & 209.43125 & -31.1054 & TW Cen & 128 & 267 & 273 \\
KG-47 & 209.43687 & -31.0558 & TW Cen & 54 & 267 & 273 \\
KG-48 & 209.77937 & -25.8551 & FQ Hya & 28 & 179 & 177 \\
KG-52 & 219.36920 & -20.3208 & LY Lib & 28 & 289 & 287 \\
KG-55 & 302.31581 & -57.3415 & BR Pav & 36 & 251 & 250 \\
KG-56 & 302.31626 & -57.3173 & BR Pav & 52 & 252 & 250 \\
KG-63 & 322.63793 & -53.9635 & X Ind & 51 & 225 & 224 \\
KG-83 & 340.79609 & -41.5202 & DS Gru & 46 & 255 & 260 \\
KG-89 & 352.03795 & -47.4441 & RU Phe & 49 & 291 & 288 \\
KG-91 & 359.12315 & -49.7759 & R Phe & 44 & 267 & 263 \\
\hline
\end{tabular}

Notes. The columns are the KG name, 2MASS coordinates (J2000) in degrees, polluting Mira star, separation angle in arcsec between the CSS object and polluant, period of the KG object in days (from CSS), period $\mathrm{P}$ of the Mira (from GCVS; for SS Col, period is from the AAVSO VSX catalog).

SMC C-type period luminosity relation (see footnote above). We find that KG-21, KG-62, and KG-67 are at distances of $28 \pm 9$, $24 \pm 5$, and $35 \pm 4 \mathrm{kpc}$, respectively, with $Z=17,17$, and $26 \mathrm{kpc}$, respectively. The uncertainties on $D$ essentially originate from $\Delta m_{\mathrm{CSS}}=2.7,1.6$, and $0.5 \mathrm{mag}$, for $\mathrm{KG}-21, \mathrm{KG}-62$, and $\mathrm{KG}-67$, respectively.

The star KG-8 is located in Fornax and we classified the object as C-type. This star is listed as not-periodic in the nearinfrared monitoring program by Whitelock et al. (2009). In their Table 3, this star is F25006. However, a period of 196 days is clearly seen in the better quality CSS data ${ }^{3}$. Its amplitude is $\Delta m_{\mathrm{CSS}}=1.3 \mathrm{mag}$. The SMC PL relation for $\mathrm{C}$ stars leads to a distance of $140 \pm 25 \mathrm{kpc}$, which agrees with $D($ Fornax $)=$ $147 \pm 12 \mathrm{kpc}$ given by McConnachie (2012). Agreement is also found for KG-3 located in Sculptor and classified C-type. With $K_{\mathrm{s}}=12.27, P=196$ days, and $\Delta m_{\mathrm{CSS}}=1.2$, our method yields $D=75 \pm 13 \mathrm{kpc}$, which is compatible with $D$ (Sculptor) $=86 \pm$ $6 \mathrm{kpc}$ from McConnachie (2012).

These agreements on external galaxies supports the view that the distances of KG-2, KG-21, KG-62, and KG-67 estimated above are plausible. This means that they are largely out of the Galactic disc, and thus we consider them to be in the halo. Our distances imply heights from the Galactic plane of between 10 and $26 \mathrm{kpc}$, much larger than $1 \mathrm{kpc}$ seen for cool $\mathrm{C}$ stars of the disc (Abia et al. 2020).

\subsubsection{M stars}

The list of the 16 LPVs that could be classified M-type, but are in fact false variables, is given in Table 2. They are in general field dwarfs with significant proper motion, angularly located close to a polluting bright Mira.

Among the remaining 77 stars, there are five M-type stars that are angularly close or in the LMC. We checked that their periods and $K_{\mathrm{s}}$ magnitudes provide a correct distance when the Soszyński et al. PL relation for M-type LMC AGBs is applied.

3 CSS light curves can be seen on the Catalina site http://nunuku. caltech.edu/cgi-bin/getcssconedb_release_img.cgi 


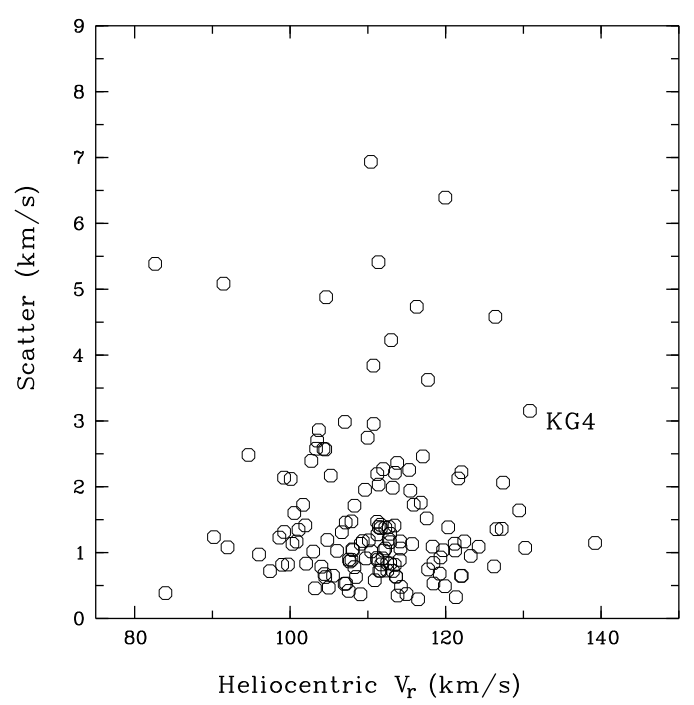

Fig. 3. APOGEE radial velocities of stars located within one degree from the centre of the Sculptor dwarf galaxy. The LPV KG-4 is indicated. Each point represents the average of 11 individual APOGEE visits. This average is plotted along the $X$ axis, and the scatter around this average is on $Y$ axis. The median error of an individual visit is only $0.18 \mathrm{~km} \mathrm{~s}^{-1}$. The high scatter for KG-4 is consistent with its variability.

These stars are KG-13, KG-15, KG-16, KG-17, and KG-18, and we obtain $50 \pm 14,58 \pm 19,53 \pm 7,51 \pm 13$, and $54 \pm 10 \mathrm{kpc}$, respectively, in good agreement with the LMC distance of $51 \mathrm{kpc}$ (adopting a distance modulus of 18.52 \pm 0.09 , from McConnachie 2012).

The star KG-4 is located at $0.345 \mathrm{deg}$ from the centre of the Sculptor dwarf galaxy, which is 1.8 times the half-light radius of this galaxy $\left(r_{h}=0.19 \mathrm{deg}\right.$, from McConnachie 2012). This star is M-type according to our examination of HES plates, and the Catalina database suggests $P=91 \mathrm{~d}, \Delta m_{\mathrm{CSS}}=0.5 \mathrm{mag}$. However, the ASAS-SN survey (Shappee et al. 2014; Jayasinghe et al. 2018) offers a slightly better light curve and a comparable time span, and finds KG-4 to be aperiodic. If we apply the SMC PL relation for M-type stars as explained below, we find $D=$ $65 \pm 7 \mathrm{kpc}$, smaller than, but marginally compatible with the distance of Sculptor $86 \pm 6$ (McConnachie 2012). Its radial velocity determined by the APOGEE experiment (Jönsson et al. 2020) shows that KG-4 is member of Sculptor, and the important velocity scatter of individual visits is consistent with its pulsating variability (Fig. 3).

The next finding of this section is the identification of five M-type stars (KG-19, KG-23, KG-72, KG-88, and KG-93) that are faint $\left(m_{\mathrm{CSS}} \sim 15\right)$, with $P$ in the range 140-240 days and $\Delta m_{\mathrm{CSS}}>0.8 \mathrm{mag}$. Their CSS names are CRTS J044714.7622118, CRTS J054202.5-553828, CRTS J220821.6-291754, CRTS J230418.1-405245, and CRTS J235855.3-430054, respectively. We obtain their distances by adopting the SMC periodluminosity relation for M-type stars from Soszyński et al. (2007):

$M_{K_{\mathrm{s}}}=-7.34-4.14 \log _{10}(P / 200)$.

Similarly to $\mathrm{C}$ stars above, this $\mathrm{SMC}$ relation is chosen because the mean SMC metallicity $([\mathrm{Fe} / \mathrm{H}]=-0.75$ to -1.0$)$ coincides with the average metallicity distribution of the Sgr tidal arms and because many of our stars can be members of these arms. Therefore, we apply relation (1), which implies distances of 50-64 kpc. If we had adopted a LMC PL relation, the distances would be systematically (but only) $10 \%$ smaller. The main characteristics of these five stars are in Table 3.

The third result of this section is that among the LPVs that could be spectrally classified, there are none fainter than $K_{\mathrm{s}}$ $0=12.5$. We did find fainter stars that could be seen and classified M, but their variability is spurious (see Mauron et al. 2019a for details), or they are members of dwarf galaxies as indicated. This lack of faint halo LPVs could be due to an instrumental limitation. For example, identifying faint LPVs may have been difficult in the process of making the CSS catalogue of Drake et al. (2017) that forms the basis of our study. Also, spectral classification is difficult or impossible for faint stars. Alternatively, it could be due to a strong decrease in the LPV number with increasing distance beyond $\sim 30 \mathrm{kpc}$. Consequently, in the following section, we focus on the CSS LPVs that lie in the HES area, but could not be classified C or M.

\subsection{Stars without $M / C$ classification from the HES}

There are 35 LPVs that could not be classified because no spectrum is visible on the Hamburg plates or because of overlapping spectra. Their Catalina $m_{\mathrm{CSS}}$ magnitudes range from 15 to 18 . Of these 35, 7 are in Fornax, 19 can be associated with the Large Magellanic Cloud, and 6 are false faint Miras (because their light is contaminated by a true nearby, bright Mira).

Among the remaining 3 unclassified LPVs, the first (hereafter named A) has remained unnoticed in the literature so far, but is interestingly faint $\left(K_{\mathrm{s}}=14.2\right)$. The second (called B) was mentioned by Grady et al. (2019) among their six most distant stars, and could be at $\sim 100 \mathrm{kpc}$. These two stars are discussed below. The CSS names of A and B are CRTS J050449.9-401314 and CRTS J135224.2-314452. Their properties are given in Table 3. Finally, the third star is a confirmed halo C-type star (2MASS J22065366-2506282) with $m_{\mathrm{CSS}}=14.07, K_{\mathrm{s}}=8.95$, $P=328$ days, and named HG-111 by Huxor \& Grebel (2015). It is possible that this star escaped our classification because a deep minimum occurred when the HES plate was exposed.

To conclude, when considering the 35 LPVs lying in the area of the Hamburg survey but not classified $\mathrm{M}$ or $\mathrm{C}$, we find only two interesting LPVs, stars A and B listed in Table 3.

\subsection{All other stars from the CSS catalogue}

In the preceding sections, the stars $\mathrm{A}$ and $\mathrm{B}$ are faint $\left(K_{\mathrm{s}}=13.6\right.$ and 14.2) and presumably distant, at about $100 \mathrm{kpc}$ if the PL relation applies. Here, we present the extension of our search to all CSS LPVs, and not only those in the HES imprint. We first noticed one peculiar object, 2MASS J15204636-2533017, with $l=340^{\circ}, b=+26^{\circ}, m_{\mathrm{CSS}}=14.93, K_{\mathrm{s}}=11.70, J-K_{\mathrm{s}}=1.01$. Its period is 323 days, and $D \sim 90 \mathrm{kpc}$ if the $K_{\mathrm{s}}$-band PL relation is valid $\left(M_{\mathrm{Ks}}=-8.2\right)$. However, a strong indication that it is closer to us is its proper motion of $1.4 \pm 0.1 \mathrm{mas} \mathrm{yr}^{-1}$, which implies a relatively high tangential velocity $\left(V_{\mathrm{t}}\right)$ of $640 \mathrm{~km} \mathrm{~s}^{-1}$ if the large distance is correct ${ }^{4}$. In addition, the light-curve amplitude is rather small, $\Delta m_{\mathrm{CSS}}=0.32 \mathrm{mag}$. Therefore, this star is likely not a pulsating AGB. It might be an ellipsoidal red giant variable similar to those of the LMC, which are 2.5 mag less luminous than AGB stars (see Fig. 2 of Soszyński et al. 2007).

To find very distant LPVs, we selected objects with $K_{\mathrm{s} 0}>12.5$ and obeying $\left(m_{\mathrm{CSS}}-K_{\mathrm{s}}\right)>2.0$ as our 77 sample stars do. We found 24 stars: eight are false variable stars, one is in Sculptor, and nine are in Fornax. Of the six remaining

\footnotetext{
${ }^{4} V_{\mathrm{t}}\left(\mathrm{km} \mathrm{s}^{-1}\right)=4.75 D(\mathrm{kpc}) \times \mu\left({\left.\text { mas } \mathrm{yr}^{-1}\right) .}^{-}\right.$
} 
N. Mauron et al.: Searching cool pulsating giants in the Galactic halo

Table 3. Properties of our candidate, distant CSS LPV stars (see text for their selection).

\begin{tabular}{|c|c|c|c|c|c|c|c|c|c|c|c|c|c|c|}
\hline Name & $\alpha$ & $\delta$ & $l$ & $b$ & $m_{\mathrm{CSS}}$ & $P$ & $\Delta m_{\mathrm{CSS}}$ & $K_{\mathrm{s}}$ & $J-K_{\mathrm{s}}$ & $A_{\mathrm{V}}$ & $D_{\mathrm{PL}}$ & $\delta D_{\mathrm{PL}}$ & $\mu$ & $V_{\mathrm{t}}$ \\
\hline KG-19 & 71.811385 & -62.355530 & 272 & -38 & 15.13 & 139 & 0.78 & 11.82 & 1.03 & 0.10 & 50 & 7 & 1.85 & 440 \\
\hline KG-23 & 85.510835 & -55.641182 & 263 & -32 & 15.65 & 167 & 1.28 & 11.65 & 1.08 & 0.15 & 54 & 10 & 1.51 & 390 \\
\hline KG-72 & 332.090176 & -29.298389 & 19 & -54 & 15.34 & 160 & 1.20 & 11.79 & 0.99 & 0.05 & 56 & 10 & 1.00 & 240 \\
\hline KG-88 & 346.075719 & -40.879162 & 354 & -64 & 14.95 & 141 & 1.27 & 12.29 & 0.77 & 0.03 & 63 & 12 & 1.26 & 380 \\
\hline KG-93 & 359.730982 & -43.014912 & 333 & -71 & 15.15 & 235 & 1.56 & 11.41 & 0.87 & 0.03 & 64 & 14 & 0.75 & 230 \\
\hline A & 76.208748 & -40.221203 & 244 & -37 & 17.44 & 108 & 0.55 & 14.16 & 1.06 & 0.08 & 119 & 14 & 0.26 & 150 \\
\hline $\mathrm{C}$ & 141.992615 & -34.560715 & 262 & +12 & 17.33 & 139 & 1.30 & 13.61 & 1.20 & 0.53 & 112 & 21 & 0.07: & 40: \\
\hline D & 154.438690 & -29.276445 & 267 & +23 & 16.36 & 184 & 0.45 & 12.68 & 1.11 & 0.20 & 93 & 10 & 0.29 & 130 \\
\hline B & 208.101207 & -31.747896 & 318 & +29 & 16.92 & 128 & 1.50 & 13.64 & 1.02 & 0.18 & 108 & 22 & 0.55 & 280 \\
\hline $\mathrm{E}$ & 217.840607 & -36.831627 & 324 & +22 & 15.76 & 110 & 0.80 & 12.81 & 0.88 & 0.26 & 64 & 9 & 0.72 & 220 \\
\hline
\end{tabular}

Notes. $m_{\mathrm{CSS}}$ is the CSS time-average magnitude. $P$ is the period in days. $\Delta m_{\mathrm{CSS}}$ is the CSS amplitude (peak to peak). $K_{\mathrm{S}}$ and $J-K_{\mathrm{s}}$ are from 2MASS. $A_{V}$ is the Galactic $V$-band extinction in mag, and $A_{K} / A_{V}=0.11 . D_{\mathrm{PL}}$ is the heliocentric distance in kpc derived with the PL sequence C relation for M-type (Eq. (1)). $\delta D$, in kpc, is the distance uncertainty (see text for details). $\mu$ is the Gaia EDR3 proper motion (see also Table 4). $V_{\mathrm{t}}$ is the tangential velocity in $\mathrm{km} \mathrm{s}^{-1}$ derived from $D_{\mathrm{PL}}$ and $\mu$. The KG stars are M-type. The spectral types of stars ABCDE are unknown.

Table 4. Gaia EDR3 parallaxes ( $\pi$ in mas) and proper motions (in mas $\mathrm{yr}^{-1}$ ) for our ten candidate distant stars.

\begin{tabular}{lccc}
\hline \hline Star & $\pi$ & $\mu_{\text {RA }}$ & $\mu_{\text {Dec }}$ \\
\hline KG-19 & $+0.005 \pm 0.021$ & $+1.814 \pm 0.024$ & $-0.367 \pm 0.031$ \\
KG-23 & $+0.023 \pm 0.027$ & $+1.507 \pm 0.033$ & $-0.042 \pm 0.036$ \\
KG-72 & $-0.001 \pm 0.033$ & $+0.115 \pm 0.035$ & $-0.994 \pm 0.030$ \\
KG-88 & $-0.040 \pm 0.041$ & $-0.320 \pm 0.037$ & $-1.211 \pm 0.029$ \\
KG-93 & $-0.018 \pm 0.036$ & $+0.461 \pm 0.037$ & $-0.590 \pm 0.034$ \\
A & $-0.060 \pm 0.072$ & $+0.231 \pm 0.076$ & $-0.118 \pm 0.081$ \\
C & $+0.010 \pm 0.068$ & $-0.031 \pm 0.055$ & $+0.066 \pm 0.056$ \\
D & $-0.036 \pm 0.057$ & $-0.162 \pm 0.058$ & $-0.238 \pm 0.048$ \\
B & $-0.049 \pm 0.070$ & $-0.419 \pm 0.079$ & $-0.363 \pm 0.090$ \\
E & $-0.002 \pm 0.041$ & $-0.514 \pm 0.043$ & $-0.500 \pm 0.039$ \\
\hline
\end{tabular}

Notes. No correction was added to $\pi$ because of the 0.030 mas zeropoint of parallaxes, as recommended in the case of individual stars (Arenou et al. 2018; their Sect. 4.5).

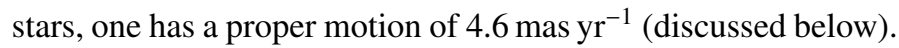
The remaining five include stars $\mathrm{A}$ and $\mathrm{B}$, as expected. The three others are referred to as C, D, and E below. The CSS names of stars C, D, and E are CRTS J092758.1-343339, CRTS J101745.2-291635, and CRTS J143121.7-364954, respectively. All five stars have proper motions of less than 0.8 mas $^{-1}{ }^{-1}$. Tables 3 and 4 give the properties of these five stars (sorted by Right Ascension).

The star with $\mu=4.6$ mas yr $^{-1}$ is 2MASS J060912004717207 and serves as another demonstration of the fact that the PL relation is not always valid. This star has an amplitude of $\Delta m_{\mathrm{CSS}}=1.4 \mathrm{mag}$. Its magnitudes are $m_{\mathrm{CSS}}=18.14$ and $K_{\mathrm{s}}=14.3$. The light curve is periodic with $P=116$ days. If it was a classical cool semi-regular, its period would put it at $125 \mathrm{kpc}$, but its proper motion would imply an excessively large tangential velocity of $\sim 2700 \mathrm{~km} \mathrm{~s}^{-1}$. Therefore, it is more probable that its distance is about ten times smaller. This star is underluminous with respect to the main (sequence $\mathrm{C}$, i.e. fundamental mode) classical PL relation.

\section{Discussion}

\subsection{Catalogue}

We provide a catalogue of the 77 LPV stars that were spectrally classified on the HES plates. They obey $\delta<-20^{\circ}$, and almost all obey $|b|>30^{\circ}$. This latitude limit is due to the HES imprint, although KG-25, KG-26, KG-40, and KG-54 are between $b=-26^{\circ}$ and $b=-29^{\circ}$, and KG-30 is at $b=+22^{\circ}$. An abridged version of the first five lines is given in Table 5. We list: J2000 coordinates, Catalina mean magnitude, period, CSSband amplitude, $K_{\mathrm{s}}(2 \mathrm{MASS})$, flag, distance in kpc, uncertainty on distance, star denomination KG-nn, M or C type, and a comment. The flag is set to 1 for normal halo LPV cases. The flag is $>1$ for members of dwarf galaxies or other peculiarities. The distances of the halo LPVs are in the range 3-65 kpc, and their heights above or below the Galactic plane are more than $5 \mathrm{kpc}$ for 57 of them.

Our list can be compared to that of Grady et al. (2019). After selecting stars with $\delta<-20^{\circ}$ and $|b|>30^{\circ}$ in the Grady list, we obtain 34 objects. Inspecting this sample shows that 8 are in the LMC, 2 are in Fornax, 1 is in Sculptor, 2 are false variables $^{5}$. There are 14 are in common with our sample, and finally, 7 remain that are not in our list. These 7 are not within the HES plates or impossible to classify with certainty. Inversely, of our initial 93 stars in the same sky imprint, we eliminate 16 false variable, 7 in the LMC, 2 in Sculptor, 1 in Fornax. Consequently, this cleaning procedure results in samples of 21 and 67 stars from the Grady list and our selection, respectively.

The main reason for which the Grady sample is smaller than ours is that these latter authors apply an amplitude cut, favouring high-amplitude variables that preferentially obey the sequence-C Mira PL relation. In contrast, the stars we seek and find are generally periodic, low-amplitude $\mathrm{SRa} / \mathrm{SRb}$ variables. Grady et al. adopt the definition of Gaia amplitude introduced by Deason et al. (2017): $\left.A_{\text {Gaia }}=\sqrt{(} N_{\text {obs }}\right) \times\left(\sigma_{F G} / F G\right)$ where $N_{\text {obs }}$ is the number of observations and $F G$ is the $G$-band flux. Also, when building their sample, Grady et al. require $\log \left(A_{\text {Gaia }}\right)>-0.55$. However, this cut discards numerous interesting stars for us. To illustrate this point, we first consider all Miras and periodic SRa and SRb of the General Catalogue of Variable Stars (Samus et al. 2017) and we plot the histogram of $\log \left(A_{\text {Gaia }}\right)$ (Fig. 4). It can be seen that the large majority (73\%) of periodic $\mathrm{SRa} / \mathrm{SRb}$ variables is missed with the Grady amplitude cut.

5 CRTS J213033.1-535749 at $\alpha=322.6379$ deg., $\delta=-53.9635$ deg., $m_{\mathrm{CSS}}=17.65, K_{\mathrm{s}}=15.6, \mathrm{P}(\mathrm{CSS})=223$ days, polluted by $\mathrm{X}$ Ind located at 51" with $\mathrm{P}(\mathrm{GCVS})=225 \mathrm{~d}$; and CRTS J224311.1-413113 at $\alpha=$ $340.7961, \delta=-41.5202, m_{\mathrm{CSS}}=16.85, K_{\mathrm{s}}=15.2, \mathrm{P}(\mathrm{CSS})=255 \mathrm{~d}$, polluted by DS Gru at $46^{\prime \prime}$ with $\mathrm{P}(\mathrm{GCVS})=260 \mathrm{~d}$. 
Table 5. Catalogue of Catalina southern LPVs with spectral classification and distances.

\begin{tabular}{lccccccccccc}
\hline \hline$\alpha(\mathrm{deg})$ & $\delta(\mathrm{deg})$ & $m_{\mathrm{CSS}}$ & $P(\mathrm{~d})$ & $\Delta m_{\mathrm{CSS}}$ & $K_{\mathrm{S}}$ & Flag & $D(\mathrm{kpc})$ & $\delta D$ & Name & Spect. & Note \\
\hline 3.1427 & -22.9213 & 12.93 & 171.1 & 1.93 & 9.22 & 1 & 16 & 4 & KG-01 & M-type & \\
4.2324 & -44.0113 & 14.71 & 440.1 & 2.40 & 7.07 & 1 & 13 & 4 & KG-02 & C-type & \\
14.9956 & -33.4764 & 16.23 & 196.0 & 1.07 & 12.27 & 6 & 75 & 12 & KG-03 & C-type & in Sculpt \\
15.3368 & -33.8847 & 16.18 & 91.5 & 0.75 & 13.14 & 6 & 59 & 8 & KG-04 & M-type & in Sculpt ? \\
15.4728 & -65.3065 & 15.71 & 162.3 & 0.50 & 12.09 & 3 & 59 & 7 & KG-05 & C-type & C in LMC \\
\hline
\end{tabular}

Notes. The full table is available at the CDS.

The same conclusion is reached when we consider $135 \mathrm{M}$ type pulsators of the Sagittarius leading arm ${ }^{6}$. These stars satisfactorily obey the main PL relation. Again, the Grady cut would eliminate more than half of these Sgr arm M-type pulsators. Consequently, we chose not to apply this Gaia amplitude selection here, but we use Gaia parallaxes and proper motions (via tangential velocities) wherever possible to eliminate stars for which the PL distance is questionable.

\subsection{Colour comparison with AGBs of globular clusters}

The colours of our halo LPVs can be compared to those of globular clusters. The Gaia-2MASS colour $G_{\mathrm{RP}}-K_{\mathrm{s}}$ is adopted here because it offers sufficient wavelength baseline, and because $G_{\mathrm{RP}}$ is the Gaia filter for which amplitudes are the lowest, compared to $G$ or $G_{\mathrm{BP}}$. In addition Gaia provides homogeneous photometry for both our LPV sample and the globular cluster stars. The interstellar extinction for the $G_{\mathrm{RP}}$ passband is $1.83 E(B-V)$ (Wang \& Chen 2019). We first consider the four metal-poor clusters M2, M3, M5, M13 at metallicities $[\mathrm{Fe} / \mathrm{H}]$ of $-1.65,-1.50,-1.29$ and -1.53 , respectively. For these clusters, García-Hernández et al. (2015) found a total of 44 AGB stars of first or second generation. The colour histograms of Fig. 5 show that the LPVs studied here are almost all redder than those of these four clusters.

A complementary view is obtained by plotting colours versus periods when periods are available (Fig. 6). We consider two globular cluster possessing a substancial number of identified LPVs: $\omega$ Cen with $[\mathrm{Fe} / \mathrm{H}]=-1.60$ and with 15 known red, slow variable stars (Lebzelter \& Wood 2016), and 47 Tuc $([\mathrm{Fe} / \mathrm{H}]=-0.70)$ with 38 variables (Lebzelter \& Wood 2005). Figure 6 shows that the majority of the cluster LPVs have $P<$ 100 days, with a few located on a rising band with larger periods and a redder colour. We note that most of our halo LPVs, including the ten distant stars (except one), are located on this band, suggesting that many cluster-like LPVs with periods in the range $\sim 30$ to 100 days remain to be identified in the halo.

Figure 6 also shows that the large-period small-amplitude LPVs seen in Fig. 2 and plotted with triangles in Fig. 6 are clearly separated from the other 'normal' LPVs. Because they are relatively blue for their periods, we suspect that those stars do not obey the P-L relation, in a way similar to the variable V13 of 47 Tuc in its long-period mode (Fig. 14 of Lebzelter \& Wood 2005). Therefore, in our catalogue, distances for these stars are not given.

\footnotetext{
6 We select them with $B_{\mathrm{Sgr}}$ between $-15^{\circ}$ and $+15^{\circ}$, and $\Lambda_{\mathrm{Sgr}}<180^{\circ}$. They can be seen in Fig. 4 of Mauron et al. (2019b) (centre panel) along the sinusoid line. The definition of longitude and latitude with respect to the Sgr stream plane is given by Belokurov et al. (2014).
}
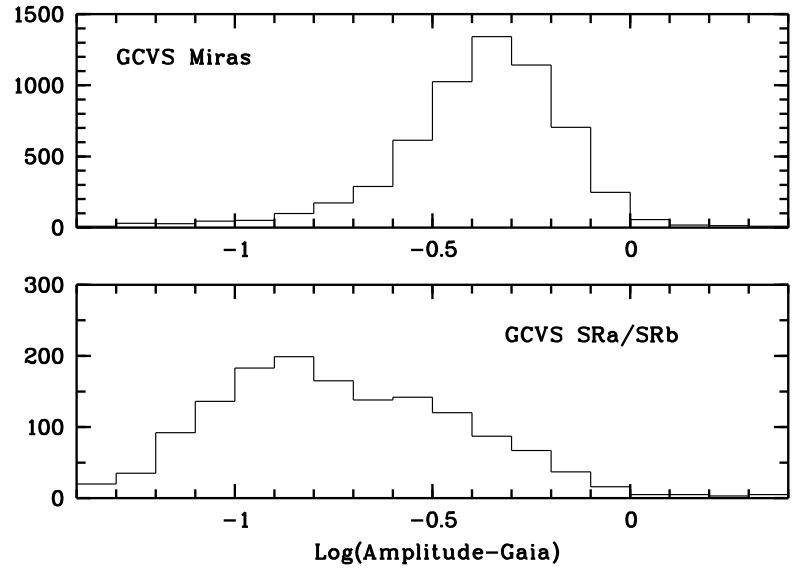

Fig. 4. Histograms of the Gaia DR2 amplitude for Mira and $\mathrm{SRa} / \mathrm{SRb}$ periodic variables of the General Catalogue of Variable Stars (Samus et al. 2017). The Gaia amplitude has been defined by Deason et al. (2017) and combines the Gaia G flux, its fluctuations, and the number of measurements (see Sect. 3.1, 2nd paragraph). The Gaia amplitude histogram is plotted in log here.

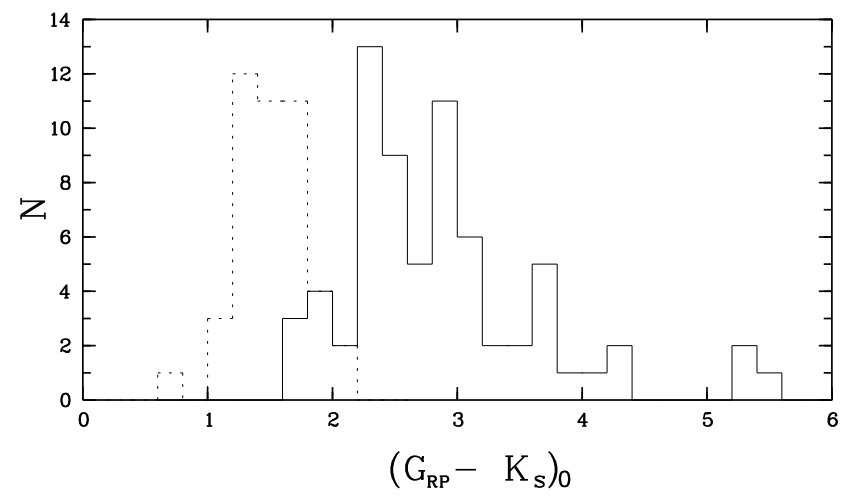

Fig. 5. Histograms of the colour $\left(G_{\mathrm{RP}}-K_{\mathrm{s}}\right)$, corrected for interstellar extinction, for the M-type halo LPVs (continuous line), and for the LPV of metal-poor globular clusters M2, M3, M5, M13, whose metallicities are between -1.3 to -1.65 (dotted line).

\subsection{Distant stars}

In Tables 3 and 4, we identified ten cases, with amplitudes $\Delta m_{\mathrm{CSS}}>0.45 \mathrm{mag}$ and $K_{\mathrm{s}}$ between 11 and 14 . These objects are considered as best candidate distant AGBs, the major finding of this study. However, the applicability of the PL relation in this case remains debatable. If this is the case, distances between 55 and $120 \mathrm{kpc}$ are derived. Table 4 shows that their Gaia DR2 proper motions are typically smaller than $\sim 1$ mas yr$^{-1}$. This translates to plausible tangential velocities of less than $\sim 450 \mathrm{~km} \mathrm{~s}^{-1}$. In Fig. 7, we plot these ten tangential velocities as a function of distance from the Sun (filled circles). 


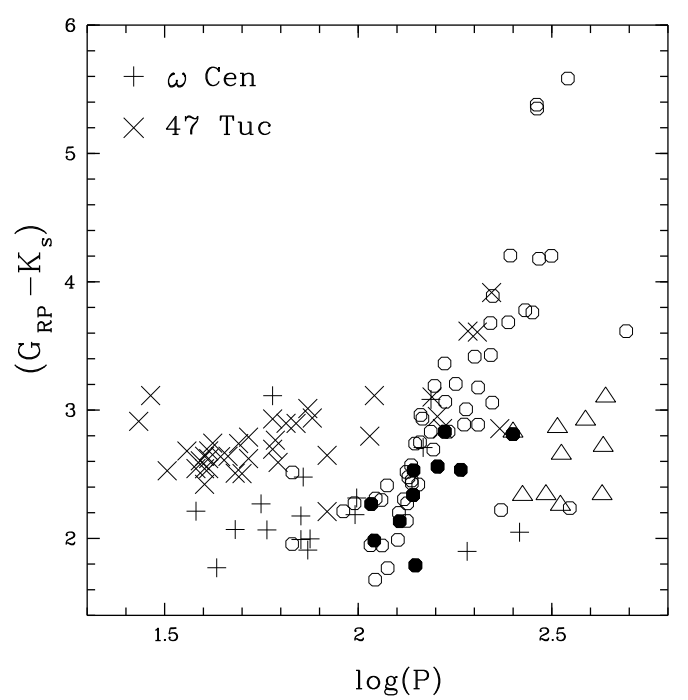

Fig. 6. Colour $\left(G_{\mathrm{RP}}-K_{\mathrm{s}}\right)$, corrected for interstellar extinction, plotted as a function of pulsation period. Circles represent the M-type LPVs of our sample. Triangles are the large-period small-amplitude group in our sample seen in Fig. 2. Filled circles are the discovered distant LPVs listed in Table 3. The LPVs of the globular clusters $\omega$ Cen $([\mathrm{Fe} / \mathrm{H}]=-1.6)$ and $47 \mathrm{Tuc}([\mathrm{Fe} / \mathrm{H}]=-0.70)$ are shown for comparison, and are indicated by plus signs and crosses, respectively. Note that a few GC LPVs with $\log (P)>2.1$ are located along the rising red main group. Uncertainties are comparable to symbol size.

For comparison, we also plot two other families of objects: all Galactic GCs, and the few dwarf galaxies for which Gaia DR2 motions have been measured. This figure shows that our candidates have reasonable $V_{\mathrm{t}}$ velocities. Most GCs have $D<20 \mathrm{kpc}$. For these, the average of their velocity $\left(\sim 250 \mathrm{~km} \mathrm{~s}^{-1}\right)$ corresponds to the reflex circular velocity of the Sun, whereas the dispersion $\left( \pm 250 \mathrm{~km} \mathrm{~s}^{-1}\right)$ reflects their intrinsic motions. The halo clusters at $D>30 \mathrm{kpc}$ have $V_{\mathrm{t}}$ between 0 and $400 \mathrm{~km} \mathrm{~s}^{-1}$. The dwarf galaxies have a similar range. In conclusion, given the large uncertainties on the small proper motions, the positions of our ten distant candidates shows them to be reasonably similar to other halo populations (clusters and dwarf galaxies), supporting their halo membership.

\subsection{An underluminous $A G B$}

We see above that 2MASS J06091200-4717207 is significantly underluminous, by approximately $5 \mathrm{mag}$. The period and amplitude of this star, 116 days and $1.4 \mathrm{mag}$, respectively, are close to or larger than those of our ten candidates. To investigate the rarity of such stars, we sought similar stars in the solar neighbourhood by considering the General Catalogue of Variable Stars (Samus et al. 2017). We required Gaia parallaxes of excellent quality, that is $\pi / \delta \pi>10$, and we found several underluminous cases. One example is CO Sge, an M-type Mira with $P=190 \mathrm{~d}$ and $\Delta V=3.6 \mathrm{mag}$, which is confirmed by the light curve of the ASAS-SN survey (Shappee et al. 2014; Kochanek et al. 2017). Its parallax is $\pi=3.45 \pm 0.18$ mas, and $D=290 \mathrm{pc}$. The flux $K_{\mathrm{s}}=4.70 \pm 0.02$ is not saturated in 2MASS. Because of variability, the mean $K_{\mathrm{s}}$-band signal is within 1.4 mag of this magnitude $^{7}$. We derive from Gaia a mean $K_{\mathrm{s}}$-band absolute magnitude

\footnotetext{
7 We found a $K_{\mathrm{s}}$ to $m_{\mathrm{CSS}}$ amplitude ratio of $\Delta K / \Delta m_{\mathrm{CSS}} \sim 0.4$ for this kind of star (Mauron et al. 2019b, their Fig. 8). A smaller ratio, $\sim 0.15$, is given for the Johnson $V$-band for cool AGBs in Sect. 2.2 of Groenewegen (2006).
}

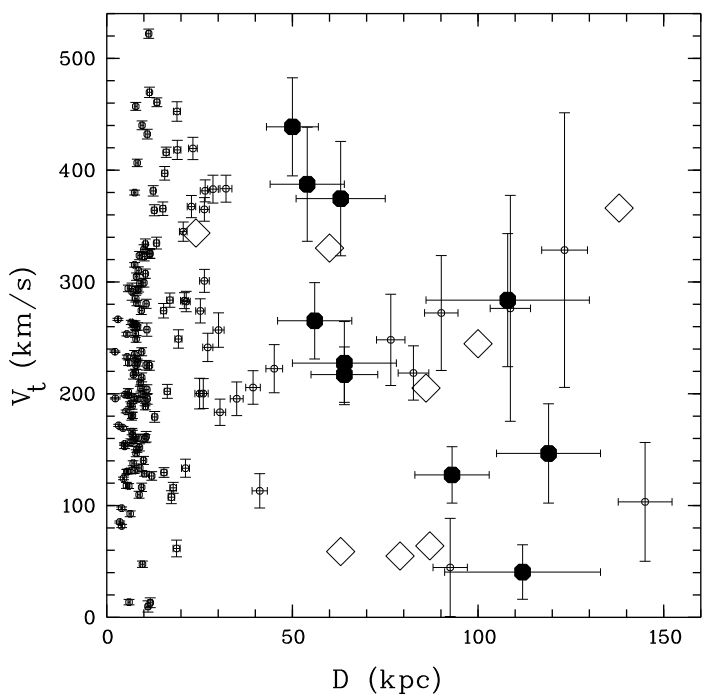

Fig. 7. Tangential velocities versus heliocentric distances. No correction for the Sun Galactic orbital motion was applied. Our ten stars in Table 3 are plotted with big filled circles. Squares are globular clusters from Harris (2010), with distance relative uncertainty of 5\%, and proper motions from Vasiliev (2019). Diamonds are dwarf galaxies: at the top, from left to right: Sgr, Boo I, Sextans, Carina, and Fornax; at the bottom, Ursa Minor, Draco, and Sculptor. Distances are from van den Bergh (2000) and proper motions from Gaia DR2 (Gaia Collaboration 2018). Errors for dwarf galaxies are comparable to the diamond size.

$M_{\mathrm{Ks}}=-2.6 \pm 1.4$, while the SMC PL relation gives -6.4 . Consequently, CO Sge is a striking example of a large-amplitude pulsator with a luminosity deficit of 3-4 mag. If our stars are similarly underluminous, their distance $D$ and velocity $V_{\mathrm{t}}$ are smaller than proposed here.

\subsection{Contaminants}

Here, we address qualitatively the issue of contamination by other halo variable stars in our sample of 77 candidate halo AGB stars. For that purpose, the very recent paper by Chen et al. (2020) (published when our work was being achieved) provides very useful information. Their work is based on the Zwicky Transient Facility (Bellm et al. 2019; Graham et al. 2019) scanning the entire northern sky in three nights, in two passbands $(g, r)$, and to magnitude $r \sim 20.5(5 \sigma)$; its Data Release 2 spans over 470 days. Chen et al. (2020) analyse the DR2 light curves and provide a catalogue of $\sim 800000$ periodic variables. They classify Mira and SR stars with periods $P>80$ days for Miras and $P>20$ days for SRs, together with cuts in amplitude, with $\operatorname{Amp}(g)>2.4$ for Miras and $\operatorname{Amp}(g)<2.4$ for SRs. These latter authors find as many as 12000 Miras and 120000 SRs, in part because ZTF covers the Galactic plane.

It is clear from their Fig. 6, which shows luminosity versus period, that a small number of binaries (classified "EW" by them) approach the locus of SRs. Most of these binaries have periods of 0.1-1days, but some are found with $P \sim$ $100 \mathrm{~d}$. Their light shapes obey characteristic relations linking the Fourier coefficients, and these relations are used to identify them (Table 1 of Chen et al.). Given that Catalina photometric quality is poorer than that of ZTF, it is very plausible that binaries contaminate the halo SR populations in which we have searched for candidates. For example, in Fig. 11, panel b, of Chen et al. (2020) the top two binary "EW" ZTF curves are quite similar 
to the two ZTF SRs shown at the top of their Fig. 12, panel a. If observed with Catalina, the curves would appear almost identical.

In conclusion, our halo LPVs may be contaminated by some binary variables, but this should improve in the future by exploiting the modern ZTF survey that surpasses Catalina in many aspects that are useful to separate true halo AGBs from these contaminants, particularly for small amplitudes. We also note that, for the region $\delta<-20^{\circ}$ not covered by ZTF, the Catalina survey has presently accumulated unique data over more than 16 years and is ongoing, and this will offer even better light curves.

\section{Summary and conclusions}

To search for AGB stars in the outer halo $(>50 \mathrm{kpc})$, we started with the CSS catalogue of LPV of Drake et al. (2017) (with $\delta<-20^{\circ}, 10000 \mathrm{deg}^{2}$, limiting magnitude $\sim 17-18$, time span 8 years). By focussing first on the CSS LPVs that are located within the imprint of the southern Hamburg-ESO objective prism plates $\left(|b|>30^{\circ}\right)$, we were able to classify (M or C) 77 of them, after discarding 16 artefacts. The large majority are Mtype. Only two new, uncatalogued $\mathrm{C}$ stars were found. After excluding members of known dwarf galaxies, all are found to be brighter than $K_{\mathrm{s}}=12.5$. This could be due to the scarcity of the sought distant objects, or to some instrumental limitation.

To search for fainter, even more distant LPVs, we considered CSS stars in the whole Drake et al. catalogue, and required $K_{\mathrm{s}}>12.5$. After discarding again false variable sources and LPVs located in the LMC, Fornax, and Sculptor dwarf galaxies, we find five candidate distant stars. In our small sample of ten objects (Table 3 ), amplitudes $\Delta m_{\text {CSS }}$ are in the range 0.45 $1.5 \mathrm{mag}$, and periods are between 100 and 230 days. We assume that the fundamental mode PL relation can provide their distance, and that they are similar to semi-regulars of the SMC. This system was chosen for its supposed low metal abundance comparable to that of our halo sample. The derived distances are found to be between $\sim 50$ and $120 \mathrm{kpc}$; their Gaia parallaxes are not significant, and their tiny proper motions are compatible with large distances and Galactic halo membership. These ten candidates are the result of this research. In addition, a catalogue of the $77 \mathrm{M}$-type and C-type stars will be made available at the CDS. During our analysis, we also found several cases of underluminous LPVs that deserve attention. Finally, the halo M-type LPVs studied here ressemble the redder slow variables of globular clusters with $P>100 \mathrm{~d}$, but the more numerous LPVs with smaller periods $(P \sim 30-100 \mathrm{~d})$ that are found in clusters remain to be identified in the halo.

The superb light curves and depth of the ZTF for northern stars, the data points acquired over seven additional years by the Catalina experiment for southern stars, and the future light curves of the Large Synoptic Survey Telescope can be considered to develop this line of research. In addition, Gaia proper motions will also improve in the near future. When follow-up spectroscopy providing radial velocities is achieved, we will hopefully have greater knowledge of the population of AGB stars in the outer halo of our Galaxy.

Acknowledgements. The authors thank the anonymous referee for remarks that greatly improved the manuscript. K. H. acknowledges support for this work from the NASA ADAP program under NASA grant 18-ADAP18-228. N. M. thanks Sebastian Otero (AAVSO) for very useful comments, Henri Reboul and Denis Puy for encouragments, and the staff of LUPM for kind assistance, especially
Stéphane Nou and Lydie Le Clainche. N. M. is grateful to the Université de Montpellier and the Institut National de Physique Nucléaire et de Physique des Particules (IN2P3; Centre National de Recherche Scientifique) for very appreciated support. This work makes use of the Catalina database (California Institute of Technology, and NASA), the Two Micron All Sky Survey (U. of Massachusetts and IPAC/Caltech funded by NASA and NSF). We very much appreciated to use the on-line archive of the Hamburg-ESO (Hamburg Observatory and European Southern Observatory) and express thanks to D. Engels. We also exploited the unique data of the European Space Agency mission Gaia. Gaia data are being processed by the Gaia Data Processing and Analysis Consortium (DPAC). Funding for the DPAC is provided by national institutions, in particular the institutions participating in the Gaia MultiLateral Agreement (MLA) The Gaia mission website is https://www. cosmos. esa.int/gaia. The Gaia archive website is https://archives.esac.esa.int/gaia. Finally, we used the SIMBAD/Vizier facilities offered by and operated at CDS, Strasbourg, France.

\section{References}

Abia, C., de Laverny, P., Cristallo, S., et al. 2020, A\&A, 633, A135 Arenou, F., Luri, X., Babusiaux, C., et al. 2018, A\&A, 616, A17 Bellm, E. C., Kulkarni, S. R., Graham, M. J., et al. 2019, PASP, 131, 018002 Belokurov, A., Koposov, S. E., Evans, N. W., et al. 2014, MNRAS, 437, 116 Bland-Hawthorn, J., \& Gerhard, O. 2016, ARA\&A, 54, 529 Bochanski, J., Willman, B., West, A., et al. 2014, AJ, 147, 76 Carlin, J. L., Sheffield, A. A., Cunha, K., \& Smith, V. V. 2018, ApJ, 859, L10 Chen, X., Wang, S., Deng, L., et al. 2020, ApJS, 249, 18 Christlieb, N., Reimers, D., \& Wisotzki, L. 2004, Messenger, 117, 40 Clement, C. 2001, AJ, 122, 2587

Clement, C. 2017, Wide-Field Variability Surveys: A 21 st Century Perpective; EPJ Web of Conferences

Deason, A. J., Belokurov, V., Erkal, D., et al. 2017, MNRAS, 467, 2636 Deason, A. J., Erkal, D., Belokurov, V., et al. 2021, MNRAS, 501, 5964 Drake, A., Djorgovski, S. G., Catelan, M., et al. 2017, MNRAS, 469, 3688 Gaia Collaboration (Helmi, A., et al.) 2018, A\&A, 616, A12 García-Hernández, D. A., Meszaros, Sz., Monelli, M., et al. 2015, ApJ, 815, L4 Gnedin, O. Y., \& Ostriker, J. P. 1997, ApJ, 474, 223

Grady, J., Belokurov, V., \& Evans, N. W. 2019, MNRAS, 483, 3022 Graham, M. J., Kulkarni, S. R., Bellm, E. C., et al. 2019, PASP, 131, 78001 Groenewegen, M. A. T. 2006, in Why Galaxies Care About AGB Stars: Their Importance as Actors and Probes, eds. F. Kerschbaum, C. Charbonnel, \& R. F. Wing, ASP Conf. Ser., 378, 433

Harmsen, B., Monachesi, A., Bell, E. F., et al. 2016, MNRAS, 466, 1491

Harris, W. E. 2010, ArXiv e-prints [arXiv:1012 . 3224]

Helmi, A. 2008, A\&ARv, 15, 145

Hernitschek, N., Schlafly, E. F., Sesar, B., et al. 2016, ApJ, 817, 73

Huxor, A. P., \& Grebel, E. K. 2015, MNRAS, 453, 2653

Janesh, W., Morrison, H. L., Ma, Z., et al. 2016, ApJ, 816, 80

Jayasinghe, T., Kochanek, C. S., Stanek, K. Z., et al. 2018, MNRAS, 477, 3145 Jönsson, H., Holtzman, J. A., Allende Prieto, C., et al. 2020, AJ, 160, 120

Kochanek, C. S., Shappee, B. J., Stanek, K. Z., et al. 2017, PASP, 129, 104502 Lebzelter, T., \& Wood, P. R. 2005, A\&A, 441, 1117

Lebzelter, T., \& Wood, P. R. 2016, A\&A, 585, A111

Lee, K. H., Lee, H. M., Fahlman, G. G., \& Sung, H. 2004, ApJ, 128, 2838

Mauron, N., Gigoyan, K. S., Gigoyan, K. K., et al. 2019a, Astrophysics, 62, 202 Mauron, N., Maurin, L. P. A., \& Kendall, T. R. 2019b, A\&A, 626, A112

McConnachie, A. W. 2012, AJ, 144, 4

Monachesi, A., Bell, E. F., Radburn-Smith, D. J., et al. 2016, MNRAS, 457, 1419

Odenkirchen, M., Grebel, E. K., Rockosi, C. M., et al. 2001, ApJ, 548, L165

Samus, N. N., Kazarovets, E. V., Durlevitch, O. V., et al. 2017, Astron. Rep., 61, 80

Schlafly, E. F., \& Finkbeiner, D. P. 2011, ApJ, 737, 103

Sesar, B., Hernischek, N., Mitrović, S., et al. 2017, AJ, 153, 204

Shappee, B. J., Prieto, J. L., Grupe, D., et al. 2014, ApJ, 788, 48

Soszyński, I., Dziembowski, W. A., Udalski, A., et al. 2007, Acta Astron., 57, 201

Starkenburg, E., Youakim, K., Martin, N., et al. 2019, MNRAS, 490, 5757

Stringer, K. M., Drlica-Wagner, A., Macri, L., et al. 2021, ApJ, 911, 109

Thomas, G. F., McConnachie, A. W., Ibata, R., et al. 2018, MNRAS, 481, 5223

van den Bergh, S. 2000, The Galaxies of the Local Group (Cambridge University Press)

Vasiliev, E. 2019, MNRAS, 484, 2832

Wang, S., \& Chen, X. 2019, ApJ, 877, 116

Whitelock, P. A., Menzies, J. W., Feast, M. W., et al. 2009, MNRAS, 394, 795

Xue, X.-X., Rix, H.-W., Ma, Z., et al. 2015, ApJ, 809, 144

Yang, C., Xue, X., Li, J., et al. 2019, ApJ, 880, 65 